\title{
O Uso de Ambientes Computacionais Modelados em 3D Como Mecanismo Preparatório Para a OBA na Educação Básica
}

\author{
José Eurimar Araújo ${ }^{1}$, Rhyan Ximenes de Brito ${ }^{1}$, \\ Janaide Nogueira de Sousa Ximenes ${ }^{2}$ \\ ${ }^{1}$ Instituto Federal de Educação, Ciência e Tecnologia do Ceará (IFCE) \\ ${ }^{2}$ Faculdade IEducare (FIED) - Rua Conselheiro João Lourenço, \\ 406 - CEP 62320-000 - Tianguá - CE - Brasil \\ \{albert.einstein222325, rxbrito, nogueirajanaide\}@gmail.com
}

\begin{abstract}
Astronomy has great fascination and curiosity for being a science that deals with the sidereal universe and the celestial bodies. In this way a study was carried out with students from the 1st to 3rd year of the middle year in order to ascertain the efficacy of textit Stellarium, textit World Wide Telescope and textit Google Earth as a support resource in the preparation for the Brazilian Astronomy Olympics. Aiming to verify efficiency as facilitators of learning. The methodology was based on bibliographical research and on the case study with high school students. The results were satisfactory in relation to the learning of the contents. The study showed the importance of 3D simulations in the teaching-learning process in Physics.
\end{abstract}

Resumo. A Astronomia exerce grande fascínio e curiosidade por ser uma ciência que trata do universo sideral e dos corpos celestes. Dessa forma realizou-se um estudo com alunos do $1^{\circ}$ ao $3^{\circ}$ ano do ensino médio a fim de averiguar a eficácia de softwares como Stellarium, World Wide Telescope e Google Earth como recurso de apoio no preparatório para Olimpíadas Brasileira de Astronomia. Objetivando verificar a eficiência como facilitadores de aprendizagem. A metodologia baseou-se em pesquisas bibliográficas e no estudo de caso com alunos do ensino médio. Os resultados mostraram-se satisfatório com relação a aprendizagem dos conteúdos abordados. O estudo mostrou a importância de simulações em $3 D$ no processo de ensino-aprendizagem na Física.

\section{Introdução}

Para [Leite and Hosoume 2007], o fascínio pelo céu motivou o homem a observá-lo e criar teorias sobre o Universo desde os primórdios da humanidade. Nesse contexto fica evidente que a Astronomia é uma ciência que trata dos astros e dos fenômenos celestes que envolvem o ser humano fazendo parte da curiosidade, do senso comum e exercendo grande motivação pela busca constante de conhecimento pelos cientistas astrônomos [Scarinci and Pacca 2006].

Vale salientar que nos Parâmetros Curriculares Nacionais (PCN), no capítulo chamado "Terra e Universo" é orientado o ensino de Astronomia na educação básica, porém, é de conhecimento que isto não acontece ou não acontece de forma correta [Langhi and Nardi 2005]. 
Nesses termos vale ressaltar a importância da Olimpíada Brasileira de Astronomia (OBA) evento organizado anualmente pela Sociedade Astronômica Brasileira (SAB), cujos objetivos são: promover o estudo da Astronomia entre alunos do ensino fundamental e médio; incentivar e colaborar com os professores destes níveis para se atualizarem em relação aos conteúdos de astronomia; Fomentar o interesse dos jovens pela astronomia; Promover a difusão dos conhecimentos básicos de uma forma lúdica e cooperativa, entre outros [Canalle et al. 2000].

Dessa forma através da modelagem computacional em 3D proporcionada por softwares como o Stellarium, World Wide Telescope ou mesmo o Google Earth trará para o discente a oportunidade de lidar com imagens, tais como: estrelas cintilantes; estrelas cadentes; chuvas de meteoros; dentre outros, na perspectiva da aprendizagem significativa [Longhini and de Deus Menezes 2010].

A motivação para este trabalho está relacionada a utilização de propostas de ensino que envolvam o aluno de forma a tornar a aprendizagem mais lúdica e atrativa, principalmente quando voltada para eventos como a OBA. Este trabalho está organizado da seguinte forma: Seção 2 apresenta alguns trabalhos relacionados, Seção 3 apresenta a metodologia, a Seção 4 apresenta os resultados e discussões e a Seção 5 as considerações finais e trabalhos futuros.

\section{Trabalhos Relacionados}

Esta seção apresenta uma revisão bibliográfica de forma reflexiva sobre a utilização de diferentes softwares durante o processo de ensino e aprendizagem.

[Sousa et al. 2018] realizaram um estudo com alunos do Curso Técnico em Informática para averiguar a eficácia do Greenfoot como ferramenta de auxílio a aprendizagem de conteúdos de programação orientada a objeto em turmas de segundo ano do ensino médio, o estudo de caso evidenciou a importância da relação teoria e prática proporcionada pela utilização de softwares como mecanismo de apoio a aprendizagem de conteúdos de programação.

[Carvalho et al. 2018] verificaram a eficiência do software Algodoo como ferramenta facilitadora de ensino e aprendizagem, através de um estudo de caso realizado com uma turma de primeiro ano do ensino médio, os resultados obtidos com relação a aprendizagem com o software Algodoo, revelaram a importância da utilização de softwares como mecanismo de apoio a aprendizagem de conteúdos de Física através de simulações em $2 \mathrm{D}$.

Para [Rodrigues et al. 2017] a aprendizagem dos conceitos de lógica de programação é essencial para os cursos da área de informática, assim propôs a aplicação de alternativas lúdicas com a utilização dos softwares RoboCode, Greenfoot e Jogos de Tabuleiro no Ensino Superior, objetivando a aplicação de alternativas que facilitasse o aprendizado do aluno, visando reduzir a reprovação e evasão, bem como aumentar o engajamento, por meio de maior integração e colaboração entre os alunos.

[Longhini and de Deus Menezes 2010] apresentaram inicialmente uma discussão sobre a utilização de Objetos Virtuais de Aprendizagem (OVA) na Educação, mais especificamente, no que se refere ao seu uso em Ensino de Ciências. A partir disso, propos seis atividades de ensino planejadas a partir de um programa computacional, o Stellarium 
(versão 0.10.1). Tais atividades foram propostas na forma de situações-problema, as quais abordam temáticas relativas à Astronomia, como os movimentos e as posições do Sol, da Lua e das estrelas, assim como a localização geográfica.

\section{Metodologia}

Esta seção descreve o planejamento das oficinas, os procedimentos de coleta e análise dos dados, assim como os participantes envolvidos durante a pesquisa e os procedimentos para coleta e análise dos dados, não necessáriamente nessa ordem.

\subsection{Planejamento das Oficinas}

As oficinas foram realizadas através de 6 encontros totalizando 6 horas, com o intuito dos participantes compreenderem sobre a história da Astronomia, Movimentos da Terra e Estações do Ano, Sistema de Coordenadas Geográficas, Planetas: Escalas, Tamanhos e Distâncias, Eclipes, Fases Lunares e Suas Influências na Terra, Leis dos Movimentos Planetários (Lei Da Gravitação e de Kepler) assim desenvolverem as competências necessárias para resolverem problemas direcionadas à OBA.

A Tabela 1 mostra a visão geral da oficina, com seus objetivos, metodologia, conteúdos, participantes, local e período.

Tabela 1. Visão Geral das Oficinas

\begin{tabular}{ll}
\hline & \multicolumn{1}{c}{ Plano de Ação } \\
\hline Objetivos & $\begin{array}{l}\text { Definir conceitos como: Dia e noite; Estações do ano; Planetas; Constelações; } \\
\text { Rotação, translação e precessão. } \\
\text { Compreender como os corpos celestes se movem e como simular isso nos } \\
\text { softwares Stellarium e Word Wide Telescope. } \\
\text { Analisar escalas e comparar os tamanhos reais de cada corpo com o auxílio } \\
\text { dos softwares Stellarium e Word Wide Telescope }\end{array}$ \\
Metodologia & $\begin{array}{l}\text { Aulas expositivas com o auxilio dos softwares Stellarium e Word Wide } \\
\text { Telescope em ambiente laboratorial ou em sala de aula }\end{array}$ \\
Conteúdo & $\begin{array}{l}\text { História da Astronomia; Movimentos da Terra e estações do ano; Sistema de } \\
\text { coordenadas geográficas; Planetas: Escalas, tamanhos e distancias ; Lua: }\end{array}$ \\
& $\begin{array}{l}\text { eclipses, fases lunares e suas influências na Terra; Leis dos movimentos } \\
\text { planetários (Lei da Gravitação e de Kepler). }\end{array}$ \\
\hline Participantes & 18 alunos do $1^{\circ}, 2^{\circ}$ e $3^{\circ}$ Ano do ensino médio \\
\hline Local & Laboratório de Informática da Escola Estadual de Ensino Profissionalizante \\
Período & Marta Maria Giffonide Sousa - Acaraú - CE
\end{tabular}

A Tabela 2 evidencia o planejamento proposto para a realização das oficinas, com o local, tempo estimado de cada encontro, conteúdo e ação a ser realizada.

\subsection{Participantes Envolvidos}

As aulas das oficinas contaram com a participação de 18 alunos das três séries do ensino médio da Escola Profissional da cidade Acaraú-CE, todos sem conhecimento sobre a manipulação das ferramentas utilizadas. 
Tabela 2. Planejamento das Atividades

\begin{tabular}{|c|c|c|c|}
\hline Local & Duração & Conteúdo & Ação \\
\hline $\begin{array}{l}\text { Laboratório de } \\
\text { Informática }\end{array}$ & $\begin{array}{l}1 \text { encontro } \\
\text { (60 } \mathrm{min})\end{array}$ & $\begin{array}{l}\text { História da } \\
\text { Astronomia }\end{array}$ & $\begin{array}{l}\text { Apresentar conceitos históricos e explanar sobre como o } \\
\text { homem via o céu e os astros, e como usavam os mesmos } \\
\text { para marcação de tempo e localização. }\end{array}$ \\
\hline $\begin{array}{l}\text { Laboratório de } \\
\text { Informática }\end{array}$ & $\begin{array}{l}1 \text { encontro } \\
\text { (60 min) }\end{array}$ & $\begin{array}{l}\text { Movimentos da } \\
\text { Terra e as estações } \\
\text { do ano }\end{array}$ & $\begin{array}{l}\text { Apresentar conceitos de rotação e translação e as estações } \\
\text { do ano de acordo com o movimento da Terra ao redor do } \\
\text { Sol. }\end{array}$ \\
\hline $\begin{array}{l}\text { Laboratório de } \\
\text { Informática }\end{array}$ & $\begin{array}{l}1 \text { encontro } \\
(60 \mathrm{~min})\end{array}$ & $\begin{array}{l}\text { Sistema de } \\
\text { coordenadas } \\
\text { geográficas }\end{array}$ & $\begin{array}{l}\text { Definir coordenadas geográficas e mostrar as mesmas com } \\
\text { o auxílio dos softwares, localizar um ponto na superfície } \\
\text { terrestre através de coordenadas terrestres. }\end{array}$ \\
\hline $\begin{array}{l}\text { Laboratório de } \\
\text { Informática }\end{array}$ & $\begin{array}{l}1 \text { encontro } \\
(60 \mathrm{~min})\end{array}$ & $\begin{array}{l}\text { Planetas: tamanhos } \\
\text { e distancias em } \\
\text { escala }\end{array}$ & $\begin{array}{l}\text { Definir o que são planetas e apresentar os planetas do } \\
\text { Sistema Solar mostrando as escalas reais de tamanhos } \\
\text { comparando-as com outros corpos do sistema solar. }\end{array}$ \\
\hline $\begin{array}{l}\text { Laboratório de } \\
\text { Informática }\end{array}$ & $\begin{array}{l}1 \text { encontro } \\
(60 \mathrm{~min})\end{array}$ & $\begin{array}{l}\text { Lua: fases lunares, } \\
\text { eclipses e } \\
\text { influências na Terra. }\end{array}$ & $\begin{array}{l}\text { Apresentar teorias de formação da Lua e suas fases, definir } \\
\text { eclipses e como eles ocorrem. }\end{array}$ \\
\hline $\begin{array}{l}\text { Laboratório de } \\
\text { Informática }\end{array}$ & $\begin{array}{l}1 \text { encontro } \\
(60 \mathrm{~min})\end{array}$ & $\begin{array}{l}\text { Movimentos } \\
\text { planetários: Lei da } \\
\text { gravitação e Leis de } \\
\text { Kepler }\end{array}$ & $\begin{array}{l}\text { Apresentar a Lei da Gravitação e as três Leis de Kepler, } \\
\text { aplicar os conceitos estudados em simulações nos } \\
\text { softwares. }\end{array}$ \\
\hline
\end{tabular}

\subsection{Softwares Utilizados}

Segue abaixo uma descrição sobre cada software utilizado no estudo de caso.

- Stellarium: Planetário de código aberto que mostra um céu realista em 3D, bastante usado em projetores de planetários, seu funcionamento está nos moldes de um planetário, com excelente qualidade técnica e gráfica é capaz de simular o céu diurno, noturno e o crepúsculo de forma muito realista [Beserra-dw et al. 2012].

- World Wide Telescope: Software desenvolvido em parceria com pesquisadores da Universidade Johns Hopkins, permite que cientistas de diversas regiões do mundo acessem imagens de objetos celestes coletadas por telescópios espaciais, com ele é possível a exploração do universo conhecido através de ambientes modelados em 3D [Jones 2013].

- Google Earth: Aplicativo que reúne diversas imagens permitindo a visualização do globo terrestre em 3D a partir de imagens obtidas por satélites de empresas como a AirPhotoUSA, Digital Globe, ESpatial, GeoEye, dentre outras [Almeida 2011].

\subsection{Procedimento de Coleta e Análise dos Dados}

Para a coleta de dados utilizada na pesquisa utilizou-se de questionários que foram aplicado ao final das oficinas realizadas com os 18 alunos. É importante destacar que os resultados foram analisados de forma estatística.

\section{Resultados e Discussões}

Os resultados contemplam três aspectos: (i) entendimento e desenvolvimento das capacidades de aprendizagem dos assuntos abordados; (ii) eficácia dos softwares softwares Stellarium, Google Earth e Word Wide Telescope relacionando teoria e prática; e (iii) eficácia do uso dos softwares Stellarium, Google Earth, e Word Wide Telescope quanto a realização, assimilação e aprendizado através das atividades propostas.

O primeiro aspecto analisado de acordo com Figura 1 foi o entendimento e desenvolvimento das capacidades de aprendizagem dos assuntos abordados nas oficinas com 
o uso dos softwares Stellarium, Google Earth e Word Wide Telescope, onde 8 alunos responderam que os softwares utilizados foram eficazes na assimilação dos conteúdos, 5 responderam que foi moderadamente eficazes, 2 acreditam que foi pouco eficazes, 1 respondeu que não foi eficaz, 2 não opinaram.

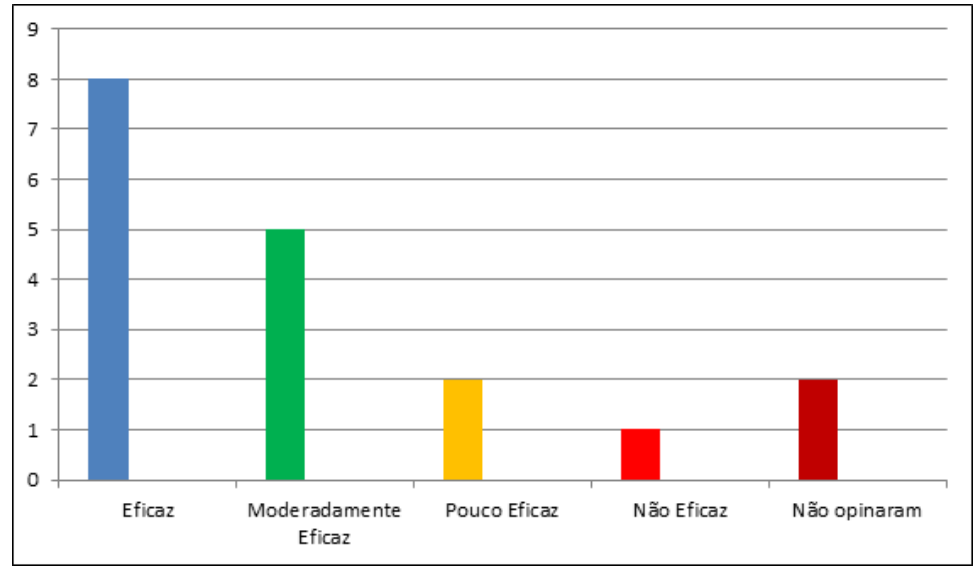

Figura 1. Assimilação com o Uso dos Softwares

O segundo aspecto analisado conforme a Figura 2 foi a eficácia na relação teoria e prática. Assim 6 alunos avaliaram como excelente a eficácia dos softwares quando relacionado teoria e prática, 3 responderam que foi muito bom, 4 acrediram que foi bom, 2 regular, 1 avaliou como insuficiente e por fim 2 não opinaram.

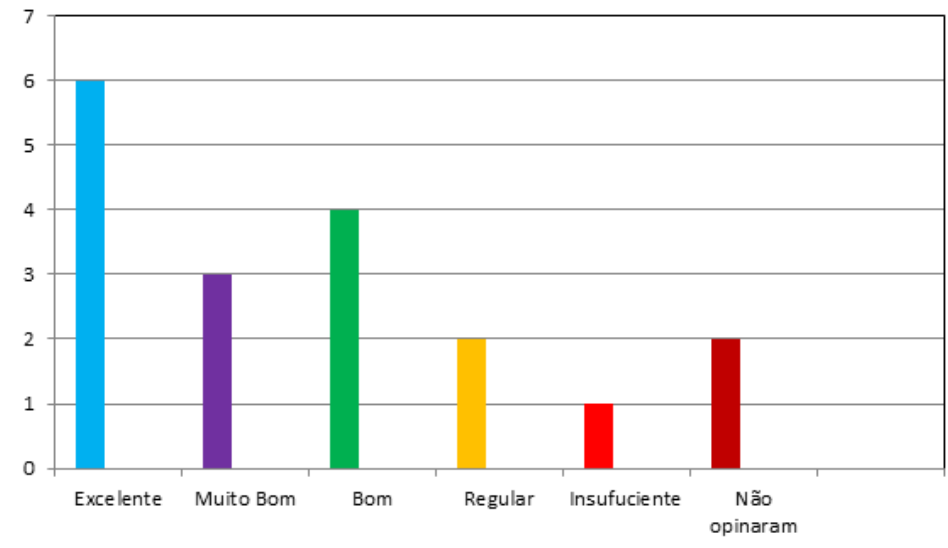

Figura 2. Eficácia dos Softwares na Relação Teoria x Prática

No terceiro aspecto conforme a Figura 3 foi avaliada a eficácia quanto a realização, assimilação e aprendizado através das atividades propostas. 7 alunos responderam que foi eficaz, 3 que foi moderamente eficaz, 5 pouco eficaz, 1 não foi eficaz e 2 não quiseram opinar.

\section{Considerações Finais e Trabalhos Futuros}

Este artigo relatou a experiência com o uso de softwares de simulação 3D como como ferramenta de ensino-aprendizagem. Comprovando-se que a utilização de ferramentas 


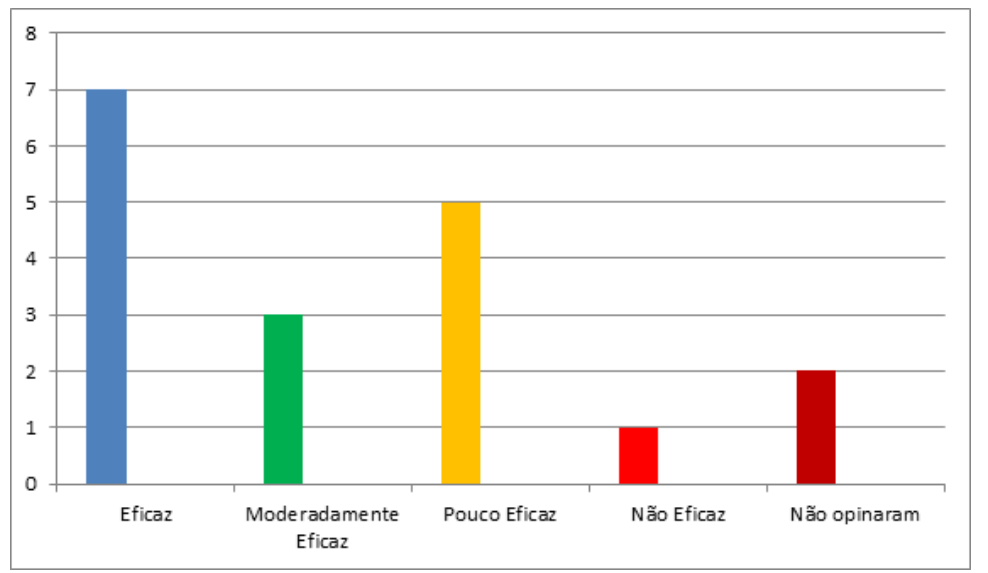

Figura 3. Eficácia do uso dos Softwares na Assimilação e Aprendizados

computacionais envolve os alunos facilitando o aprendizado de conteúdos que exijam abstração, minimizando problemas de assimilação de conteúdos de área como a Física.

Como trabalho futuro sugere-se a coleta dos dados antes e depois da ferramentas a fim de comparar os resultados obtidos de forma a avaliar os impactos na aprovação dos educandos.

\section{Referências}

Almeida, R. D. d. (2011). Novos rumos da cartografia escolar: currículo, linguagem e tecnologia. São Paulo: Contexto.

Beserra-dw, D. W. S., Barbosa, A. O., de Andrade, M. J. P., and de Araújo, A. E. P. (2012). Ensino de astronomia com os softwares stellarium e celestia.

Canalle, J. B. G., Lavouras, D. F., Arany-Prado, L. I., and de Oliveira Abans, M. (2000). Ii olimpíada brasileira de astronomia e participação na iv olimpíada internacional. $\mathrm{Ca}$ derno Brasileiro de Ensino de Física, 17(2):239-247.

Carvalho, L., Araújo, M. V., and Brito, R. (2018). Um estudo de caso com a ferramenta algodoo para o ensino da disciplina de física no ensino médio através de simulações computacionais em 2d. In Anais da IV Escola Regional de Informática do Piauí, pages 184-189. SBC.

Jones, F. (2013). Cientistas podem contar uma história sem perder a acurácia. Revista Brasileira de Cirurgia Cardiovascular/Brazilian Journal of Cardiovascular Surgery, 28(2):300-301.

Langhi, R. and Nardi, R. (2005). Dificuldades de professores dos anos iniciais do ensino fundamental em relação ao ensino da astronomia. Revista Latino-Americana de Educação em Astronomia, (2):75-91.

Leite, C. and Hosoume, Y. (2007). Os professores de ciências e suas formas de pensar a astronomia. Revista Latino-Americana de Educação em Astronomia, (4):47-68.

Longhini, M. D. and de Deus Menezes, L. D. (2010). Objeto virtual de aprendizagem no ensino de astronomia: Algumas situações problemas propostas a partir do software stellarium. Caderno Brasileiro de Ensino de Física, 27(3):433-448. 
Rodrigues, L. C., Nogueira, G. C., and Queiroga, A. (2017). Experiências no ensino de programação orientada a objetos: Robocode, greenfoot e jogos de tabuleiro no ensino superior. In Anais do Workshop de Informática na Escola, volume 23, page 598.

Scarinci, A. L. and Pacca, J. L. d. A. (2006). Um curso de astronomia e as pré-concepções dos alunos. Revista Brasileira de Ensino de Física, 28(1):89-99.

Sousa, C., Brito, R., Ximenes, J., and Silva, P. H. (2018). Estudo de caso com a ferramenta greenfoot para o ensino da programação orientada a objetos de forma lúdica na escola profissional de viçosa do ceará. In Anais da IV Escola Regional de Informática do Piauí, pages 109-114. SBC. 